\title{
MAGNETIC BUOYANCY WITH VISCOSITY AND OHMIC DISSIPATION AND FLUX TUBE FORMATION
}

\author{
V.D.KUZNETSOV \\ IZMIRAN, Troitsk, Moscow Region 142092, USSR
}

In the framework of the magnetohydrodynamic equations with dissipative terms in the form of turbulent viscosity $\left(\nu_{\mathrm{t}}\right)$ the linear stage of the instability of a subphotospheric field with respect to magnetic buoyancy is considered. For an exponential in $z$, isothermal plane-parallel atmosphere with a constant Alfvenic velocity

$$
\stackrel{2}{c_{\mathrm{S}}}=k T / m=\mathrm{const}, \quad \alpha=B_{0}^{2} /\left(8 \pi \rho_{0}\right)=v_{\mathrm{A}}^{2} / 2 c_{\mathrm{s}}^{2}=\mathrm{const}
$$

the perturbations of the form

$$
f(z) \exp \left[i \omega t+i k_{\mathrm{x}} x+i k_{\mathrm{y}} y\right]
$$

are described by differential equation with constant coefficients. The qualitative dependence of the growth rate of instability on transverse wave number is determined (see Figures 1 and 2) and characteristic scales of the magnetic tubes are evaluated at the linear stage of instability (Kuznetsov, 1987, 1988).

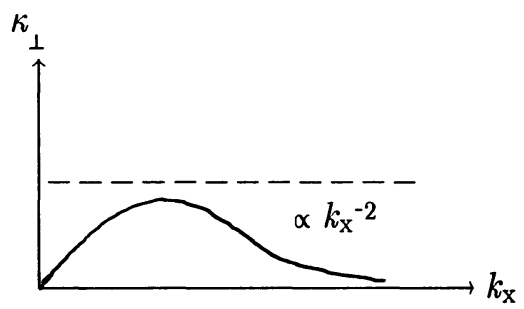

Figure 1. The case $\nu_{\mathrm{m}}=0, k_{\mathrm{y}}=0$ (kinematic viscosity)

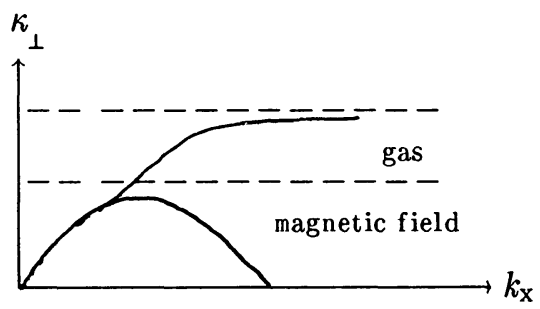

Figure 2. The case $\nu_{\mathrm{t}}=0, k_{\mathrm{y}}=0$ (magnetic viscosity)

The perpendicular wavelength is given by

$$
\lambda_{\perp} \approx 2 \pi\left(\nu_{\mathrm{t}} c_{\mathrm{s}} / g\right)^{1 / 2}=1.4 \cdot 10^{8} \mathrm{~cm}
$$

58

E. R. Priest and V. Krishan (eds.), Basic Plasma Processes on the Sun, 58-59.

(C) 1990 IAU. Printed in the Netherlands. 
where

$$
\nu_{\mathrm{t}}=6 \cdot 10^{12} \mathrm{~cm}^{2} \mathrm{~s}^{-1}, \quad H=c_{\mathrm{s}}^{2} / g=1.4 \cdot 10^{8} \mathrm{~cm} .
$$

The parallel wavelength lies in the range (Parker, 1979)

$$
2 \cdot 10^{9} \mathrm{~cm} \approx 14.5 H \approx 8 \pi H / \sqrt{3}<\lambda_{\|}<4 \pi H / \sqrt{\sqrt{2}-1} \approx 19.5 H \approx 2.7 \cdot 10^{9} \mathrm{~cm}
$$

For the above parameters characteristic of the top of the solar convective zone, the longitudinal and transverse scales of the emerging flux tubes are in good agreement with the sizes of the magnetic structures observed as arches and loops. The fragmentation of the subphotospheric magnetic field into flux tubes must be most effective in the hydrogen ionization zone where the value of the adiabatic index of the gas $\gamma$ is minimum and the elasticity of the gas cannot stabilize the instability. Taking at the depth $h=3 \cdot 10^{8} \mathrm{~cm}$ under the photosphere the parameters $p_{0}=10^{8}$ dyn $/ \mathrm{cm}^{2}, T=2.5 \cdot 10^{4} \mathrm{~K}, \gamma=1.09$ we find that for the instability of the longitudinal perturbations (Parker 1979)

$$
\gamma<\Gamma=(1+\alpha)^{2} /(1+3 \alpha / 2) \approx 1+\alpha / 2
$$

the value of the magnetic field

$$
B_{0} \geq\left[16 \pi p_{0}(\gamma-1)\right]^{1 / 2} \simeq 2 \cdot 10^{4} \mathrm{G}
$$

is needed, which is quite acceptable.

\section{References}

Kuznetsov, V.D. (1987) 'A typical sizes of the flux tubes in the unstable atmosphere with turbulent viscosity and ohmic dissipation', Magnitnaya Gidrodinamika 2, 13-18 (in Russian).

Kuznetsov, V.D. (1988) 'Magnetic field fragmentation into flux tubes in sub photospheric layers of the Sun', in: E.I.Mogilevskiy (ed.), Fizika Solnechnoy Aktivnosti, Nauka, Moscow, pp. 30-35 (in Russian).

Parker, E.N. (1979) 'Cosmical Magnetic Fields', Clarendon Press, Oxford. 